\title{
Behavioural divergence of sympatric Anopheles funestus populations in Burkina Faso
}

\author{
Wamdaogo M Guelbeogo ${ }^{1 *}$, N'Fale Sagnon ${ }^{1}$, Fang Liu², Nora J Besansky ${ }^{3^{*}}$ and Carlo Costantini ${ }^{4}$
}

\begin{abstract}
Background: In Burkina Faso, two chromosomal forms of the malaria vector Anopheles funestus, Folonzo and Kiribina, are distinguished by contrasting frequencies of shared polymorphic chromosomal inversions. Sympatric and synchronous populations of Folonzo and Kiribina mate assortatively, as indicated by a significant deficit of heterokaryotypes, and genetic associations among inversions on independently segregating chromosome arms. The present study aimed to assess, by intensive longitudinal sampling, whether sympatric Folonzo and Kiribina populations are characterized by behavioural differences in key malaria vectorial parameters.

Methods: The study was conducted in two adjacent villages near Ouagadougou, in the dry savanna of central Burkina Faso. Mosquito adult resting behaviour of both forms was compared based on parallel indoor/outdoor collections across six breeding seasons; 8,235 fully karyotyped samples of half-gravid females were analysed in total. Additionally, indoor/outdoor human biting behaviour, host selection, and Plasmodium falciparum sporozoite rate was assessed and compared between chromosomal forms.

Results: The Kiribina form was numerically predominant in the area. However, the Folonzo form was significantly over-represented in indoor resting collections and showed stronger post-prandial endophily, while Kiribina predominated outdoors. Neither form was statistically distinguishable in human biting behaviour, and both were more likely to seek human blood meals indoors than outside. The human blood index and sporozoite rate were comparably high in both chromosomal forms in indoor collections ( $>89 \%$ and $>8 \%$, respectively).

Conclusions: Both Kiribina and Folonzo chromosomal forms are formidable malaria vectors in Burkina Faso. However, the significantly greater tendency for the Kiribina form to rest outdoors despite its pronounced anthropophily suggests that uniform exposure of the overall An. funestus population to indoor-based vector control tools cannot be expected; Kiribina is more likely to evade indoor interventions and escape unharmed outdoors, reducing the efficacy of malaria control. Accordingly, more efficient methods to detect Kiribina and Folonzo, and a more complete understanding of their distribution and behaviour in Africa are advocated.
\end{abstract}

Keywords: Anopheles funestus, Anthropophily, Behavioural divergence, Chromosomal forms, Exophily, Folonzo, Kiribina, Malaria vector, West Africa

\section{Background}

The malaria vectorial system in tropical Africa is dominated by four species of major importance, Anopheles gambiae, Anopheles coluzzii, Anopheles arabiensis and Anopheles funestus, which are broadly codistributed across much of tropical Africa in close association with humans

\footnotetext{
* Correspondence: guelbeogo.cnrfp@fasonet.bf; nbesansk@nd.edu

${ }^{1}$ Centre National de Recherche et de Formation sur le Paludisme,

Ouagadougou 01 BP 2208, Burkina Faso

${ }^{3}$ Eck Institute for Global Health, Department of Biological Sciences, University of Notre Dame, Notre Dame, IN 46556, USA

Full list of author information is available at the end of the article
}

[1,2]. The first three species belong to the same cryptic species complex (the An. gambiae complex) whose members cannot be distinguished morphologically at any developmental stage, although they differ in aquatic larval ecology and adult behaviours relevant to malaria transmission and control (e.g., degree of anthropophily and tendency to blood-feed or rest indoors) [3,4]. Anopheles funestus and its presently recognized closest relatives are classified into a group and subgroup [5,6] rather than a species complex, owing to slight morphological distinctions mainly at immature stages. However, further cryptic 
taxonomic complexities within the group have recently come to light and more can be anticipated as An. funestus research emerges from a period of neglect [7-11]. Malaria transmission by the Funestus Subgroup is overwhelmingly attributed to $A n$. funestus sensu stricto, owing to its strong preference for human blood meals (see reviews by $[7,12]$ ).

Anopheles funestus s.s. is characterized by abundant genetic polymorphism, exemplified by at least 17 chromosomal re-arrangements segregating within and among populations across Africa [13,14]. Although this species is generally considered to be uniformly anthropophilic and endophilic throughout its range, complex and incompletely understood patterns of population structure based on cytogenetic and DNA markers have been detected [15-20]. In particular, two chromosomal forms designated "Folonzo" and "Kiribina" have been described in West Africa [16]. First discovered in Burkina Faso and most intensively characterized in that country, Folonzo and Kiribina populations carry markedly different frequencies of shared polymorphic chromosomal inversions, mainly involving arm 3R [16,17]. In localities where the chromosomal forms are synchronous and stably sympatric across successive breeding seasons and years, there are highly significant departures from Hardy Weinberg equilibrium and significant genetic associations among physically unlinked inversion systems; alternative homokaryotypes are more frequent than expected under random mating, and there are significant deficits of heterozygotes in virtually all population samples, consistent with assortative mating by form $[16,17]$.

Neither inversions nor inversion combinations are diagnostic taxonomic characters. However, the Kiribina form is predominantly homokaryotypic for the standard chromosomal arrangements, while Folonzo, the more chromosomally polymorphic taxon, carries high frequencies of inversions $3 \mathrm{Ra}$ and $3 \mathrm{Rb}$, and presumably corresponds to An. funestus from East Africa, where Kiribina has not been recorded [16]. Strongly reminiscent of the chromosomal forms of An. gambiae [21], these alternative karyotypes show cyclical patterns of seasonal variation in relative abundance linked to temperature and rainfall, likely reflecting differences in geographic distribution modulated by larval habitat utilization [22]. Although direct evidence is lacking, the Folonzo form is associated with natural larval habitats such as marshes, while Kiribina is associated with larval habitats created by the practice of agriculture, notably rice fields. Molecular genetic studies using mtDNA and microsatellite markers revealed very slight but significant divergence between sympatric samples of Folonzo and Kiribina across Burkina Faso, although nuclear divergence was not genome-wide and could be explained by loci on chromosome $3 \mathrm{R}$ inside and outside inversions $[23,24]$. These data are suggestive of an incipient process of ecological divergence and lineage splitting, similar to, but less advanced than, that responsible for the divergence of An. coluzzii and An. gambiae (formerly recognized as chromosomal or molecular forms $[25,26])$.

Previous studies in Burkina Faso and Senegal have reported similarly high rates of anthropophily and comparable Plasmodium falciparum infection rates in sympatric Folonzo and Kiribina populations [23,24,27]. However, there were indications of differences in indoor resting behaviour, leading to the suggestion that the Kiribina form may be more easily diverted to outdoor resting and biting, particularly in localities where alternative hosts such as cattle outnumber the local human population [16]. If the ecological and genetic heterogeneities between Folonzo and Kiribina indeed extend to behavioural differences of importance to malaria epidemiology and control, these vectorial differences must be understood more deeply. Toward that end, resting and biting behaviour were assessed separately for sympatric and synchronous Folonzo and Kiribina populations in the rural villages of Kuiti and Koubri near Ouagadougou, Burkina Faso. Observations spanned six breeding seasons and 8,235 fully karyotyped Folonzo and Kiribina adult half-gravid females.

\section{Methods}

\section{Study area}

The study was carried out in the arid Sudan savanna vegetation belt of West Africa, in adjacent rural villages located $35 \mathrm{~km}$ south of Ouagadougou, the capital of Burkina Faso. Koubri $\left(12^{\circ} 11^{\prime} 54 \mathrm{~N} ; 1^{\circ} 23^{\prime} 43 \mathrm{~W}\right)$ and Kuiti $\left(12^{\circ} 11^{\prime} 36 \mathrm{~N}\right.$; $\left.1^{\circ} 23^{\prime} 11 \mathrm{~W}\right)$ lie about $1 \mathrm{~km}$ apart on opposite margins of an artificial lake bordered by permanent swamps. A detailed map and additional information about the study area can be found elsewhere $[17,24]$. In this region, the $A n$. funestus breeding season commences at the end of the rainy season (September), extends throughout the cool dry season (October-February), and ends in April, mid-way through the hot dry season (March-May). Folonzo peaks in relative abundance following the rains, in OctoberDecember [22].

\section{Chromosomal form identification}

Adult Funestus Group females were sorted morphologically [28] in the field under a dissecting microscope. Ovaries from females at the appropriate gonotrophic stage were immediately dissected and preserved by individual mosquito in $1.5 \mathrm{ml}$ microcentrifuge tubes using Carnoy's fixative (ethanol:glacial acetic acid, 3:1), while the associated carcass was placed in a correspondingly labelled microcentrifuge tube with desiccant. Molecular taxonomic identification of An. funestus based on DNA extracted from the carcass was performed with a modified rDNA-based PCR assay [24]. Ovaries in Carnoy's were held on ice until they could be stored at $-20^{\circ} \mathrm{C}$ for later polytene chromosome analysis. Polytene chromosomes of An. funestus were spread [29] and examined 
under a phase-contrast microscope. Karyotypes were assigned using the cytogenetic map of Sharakhov et al. [13]. Of all karyotyped samples, 92\% were successfully scored for all inversions. Chromosomal form assignment followed the deterministic algorithm of Guelbeogo et al. [17]. Using a probabilistic assignment test as an alternative method of classification of karyotypes sampled from the same localities as the present study, these authors estimated the rate of mis-classification to be very low, about $0.7 \%$.

\section{Resting behaviour}

An estimate of the odds of adult females of the Folonzo or Kiribina forms resting indoors was calculated by comparing the relative abundance of each form in resting collections that were conducted indoors and outdoors in parallel. Indoor resting mosquitoes were sampled in the afternoon inside multiple huts and compounds in both villages, by insecticide spray-sheet catches (ISC) three times per week; mosquitoes resting outdoors in the villages were sampled at least twice weekly from four Muirhead-Thompson style pit-shelters with manual aspirators [30]. In addition, an estimate of the odds of postprandial indoor-resting by outdoor-biting Folonzo or Kiribina was calculated based on blood meal identifications performed on indoor/outdoor-resting collections made between 2005-2007 (described below). As cattle do not share the domestic environment with humans in the study area, mosquitoes with exclusively bovine blood meals must have fed outdoors on cattle. Accordingly, the numbers of each form that fed solely on cattle were compared between indoor-resting (ISC) and outdoor-resting (PIT) collections from the same time period.

\section{Human biting behaviour}

Human biting behaviour of Folonzo and Kiribina was assessed by human landing catches (HLC). Two teams of trained collectors worked in two different compounds in eight-hour shifts (21:00-05:00), twice per week from October 2002-January 2003. Each team consisted of a pair of collectors, one of whom performed an indoor landing catch while the other did the same outdoors, reversing positions on a subsequent night to control for collector-specific effects. To identify the chromosomal form of host-seeking An. funestus captured by HLC, mosquitoes were blood-fed on rabbits the same night of capture, and held in the insectary until they reached the stage of ovarian development appropriate for polytene chromosome analysis.

\section{Blood meal identification and Plasmodium falciparum detection}

Samples of blood-fed mosquitoes collected in the 20052006 and 2006-2007 breeding seasons were cut transversely between the thorax and the abdomen. The origin of the blood meal (human, bovine, mixed) in the posterior portion was identified by an enzyme-linked immunosorbent assay (ELISA) using specific monoclonal antibodies. The presence of $P$. falciparum circumsporozoite protein (CSP) in the anterior portion (head + thorax) also was detected by ELISA in these samples.

\section{Data analysis}

The human blood index (HBI) of each chromosomal form was calculated as the proportion of human and mixed blood meals identified relative to all blood meals identified by ELISA in samples of that form. The sporozoite rate of each form was calculated as the proportion of mosquitoes in a sample that were positive for $P$. falciparum CSP by ELISA. The odds ratio (OR; [31]), the ratio of the odds of an event occurring in one group to the odds of it occurring in another, was used to compare vectorial parameters between the chromosomal forms. The precision of the OR was estimated using the 95\% confidence interval (CI). $P$-values are reported based on the Pearson Chi-square test of association for $2 \times 2$ contingency tables, with $P<0.05$ considered as significant.

\section{Ethical approval}

The study protocols were reviewed and approved by the institutional health ethical review board of Burkina Faso. Informed consent was obtained from participants.

\section{Results}

Resting behaviour by sympatric populations of the two chromosomal forms of An. funestus was assessed by parallel indoor/outdoor collections across six breeding seasons in two adjacent rural villages located in the dry savanna of central Burkina Faso. The results from the 8,235 fully karyotyped samples are presented in Table 1 . Samples of the Kiribina form were generally larger than corresponding samples of the Folonzo form both indoors and out, particularly for the outdoor collections. However, during most breeding seasons and for the pooled samples, the Folonzo form was more likely than Kiribina to rest inside human dwellings rather than outside in pit-shelters. For $50 \%$ of the seasons, particularly when the numbers of outdoor-resting mosquitoes were sufficiently large, a Chi-square test of association for a $2 \times 2$ contingency table indicated that the stronger indoor resting tendency of Folonzo relative to Kiribina was statistically significant (Table 1).

A measure of post-prandial resting behaviour by outdoorfeeding An. funestus was estimated by focusing on those mosquitoes with exclusively bovine-derived blood meals and comparing their numbers between indoor-resting and outdoor-resting samples of each chromosomal form. Based on this measure, the Folonzo form was significantly more 
Table 1 Resting behaviour of Anopheles funestus chromosomal forms in Burkina Faso

\begin{tabular}{|c|c|c|c|c|c|c|c|}
\hline Season & Sample & Total & Folonzo & Kiribina & OR & $0.95 \mathrm{Cl}$ & $P$ \\
\hline \multirow[t]{2}{*}{$1999-2000$} & ISC/indoor & 1154 & 377 & 777 & 1.94 & $1.29-2.93$ & 0.001 \\
\hline & PIT/outdoor & 155 & 31 & 124 & & & \\
\hline \multirow[t]{2}{*}{$2000-2001$} & ISC/indoor & 1164 & 208 & 956 & 1.58 & $0.55-4.54$ & NS \\
\hline & PIT/outdoor & 33 & 4 & 29 & & & \\
\hline \multirow[t]{2}{*}{$2001-2002$} & ISC/indoor & 2733 & 659 & 2074 & 0.95 & $0.49-1.84$ & NS \\
\hline & PIT/outdoor & 48 & 12 & 36 & & & \\
\hline \multirow[t]{2}{*}{$2002-2003$} & ISC/indoor & 485 & 301 & 184 & 3.03 & $1.70-5.37$ & $<0.0001$ \\
\hline & PIT/outdoor & 57 & 20 & 37 & & & \\
\hline \multirow[t]{2}{*}{$2005-2006$} & ISC/indoor & 99 & 63 & 36 & 2.33 & $0.49-11.02$ & NS \\
\hline & PIT/outdoor & 7 & 3 & 4 & & & \\
\hline \multirow[t]{2}{*}{ 2006-2007 } & ISC/indoor & 932 & 343 & 589 & 11.87 & $8.93-15.76$ & $<0.0001$ \\
\hline & PIT/outdoor & 1368 & 64 & 1304 & & & \\
\hline \multirow[t]{2}{*}{ Pooled } & ISC/indoor & 6567 & 1951 & 4616 & 4.84 & $4.02-5.82$ & $<0.0001$ \\
\hline & PIT/outdoor & 1668 & 134 & 1534 & & & \\
\hline
\end{tabular}

OR, odds ratio of indoor/outdoor resting by Folonzo versus Kiribina; Cl, confidence interval; ISC, insecticide spray-sheet catch; PIT, Muirhead-Thomson pit-shelter [30].

likely than Kiribina to rest indoors following a bovine blood meal taken outdoors (Table 2).

HLC, conducted in parallel indoors and outdoors during the 2002-2003 breeding season, were used to compare human-biting behaviour between the chromosomal forms. Based on the $>1,000$ female mosquitoes captured, karyotyped and assigned to chromosomal form in 2002-3, human biting behaviour indoors versus outdoors was indistinguishable between chromosomal forms (Table 3). For both, the proportion of mosquitoes seeking human blood meals indoors versus outdoors was higher and of a similar magnitude. Importantly, the absolute numbers of the Folonzo form captured by HLC, both indoors and out, were larger than those from the corresponding Kiribina samples. These observations suggest that the Kiribina form may be more opportunistic, and the Folonzo form more anthropophilic, in host-seeking behaviour.

Host selection was assessed by blood meal identification during the 2005-2006 and 2006-2007 breeding seasons. The indoor resting samples of Folonzo and Kiribina both had a relatively high human blood index, 95.9 and 89.3\%, respectively (Table 4). Folonzo was the form more likely

Table 2 Post-prandial resting behaviour of outdoor-feeding Anopheles funestus chromosomal forms in Burkina Faso (2005-2007)

\begin{tabular}{lllllll}
\hline Sample* & Total & Folonzo & Kiribina & OR & $\mathbf{0 . 9 5} \mathbf{C l}$ & $\boldsymbol{P}$ \\
\hline ISC/indoor & 38 & 9 & 29 & 5.64 & $2.43-13.10$ & 0.0003 \\
PIT/outdoor & 518 & 27 & 491 & & & \\
\hline
\end{tabular}

OR, odds ratio of post-prandial indoor resting by Folonzo versus Kiribina for outdoor-feeding An. funestus (2005-2007 season); Cl, confidence interval; PIT, Muirhead-Thomson pit-shelter [30]; ISC, insecticide spray-sheet catch. *Includes only An. funestus having blood fed exclusively on cattle, based on blood meal identification. to have fed on humans in whole or in part, rather than solely on cattle $(P<0.006)$, but this trend may reflect differences between forms in post-prandial resting behaviour rather than differences in the intrinsic preference for human hosts (i.e., host choice). While the size of indoor samples was balanced between Folonzo and Kiribina, there was a large skew in outdoor resting sample size between the forms, only 30 for Folonzo compared to 529 for Kiribina, reflecting the greater tendency for the latter form to rest outdoors. Both outdoor resting samples had drastically lower human blood indices, 10 and 7\% for Folonzo and Kiribina, respectively. Folonzo remained the form more likely to have fed on humans than cattle (OR, 1.44), although this trend was not statistically significant.

During the same 2005-2007 seasons that host selection was evaluated, samples were analysed for P. falciparum infection by testing for the presence of the CSP in indoor and outdoor resting samples of the two chromosomal forms. Infection rates in the indoor resting samples did not differ significantly between forms, being similarly high in both (8.5-8.8\%; Table 5). Among the outdoor resting mosquitoes, the small Folonzo sample contained no sporozoitepositives, while the much larger Kiribina sample contained 32 (3\%) sporozoite positives.

\section{Discussion}

Intensive longitudinal sampling of An. funestus from adjacent villages in the Sudan savanna of Burkina Faso, West Africa, affirms and extends the previous findings by Costantini et al. [16] of behavioural divergence between sympatric and synchronous chromosomal forms known as Folonzo and Kiribina. The high rate of anthropophagy by both forms ( $>89 \%$ of indoor samples), 
Table 3 Human biting behaviour of Anopheles funestus chromosomal forms in Burkina Faso (2002-2003)

\begin{tabular}{|c|c|c|c|c|c|c|c|}
\hline Month & Sample & Total & Folonzo & Kiribina & OR & $0.95 \mathrm{Cl}$ & $P$ \\
\hline \multirow[t]{2}{*}{ October } & HLC/indoor & 363 & 263 & 100 & 0.87 & $0.54-1.40$ & NS \\
\hline & HLC/outdoor & 117 & 88 & 29 & & & \\
\hline \multirow[t]{2}{*}{ November } & HLC/indoor & 218 & 151 & 67 & 1.18 & $0.74-1.87$ & NS \\
\hline & HLC/outdoor & 131 & 86 & 45 & & & \\
\hline \multirow[t]{2}{*}{ December } & HLC/indoor & 70 & 42 & 28 & 0.62 & $0.30-1.30$ & NS \\
\hline & HLC/outdoor & 58 & 41 & 17 & & & \\
\hline \multirow[t]{2}{*}{ January } & HLC/indoor & 80 & 45 & 35 & 1.63 & $0.73-3.65$ & NS \\
\hline & HLC/outdoor & 34 & 15 & 19 & & & \\
\hline \multirow[t]{2}{*}{ Pooled } & HLC/indoor & 731 & 501 & 230 & 1.04 & $0.79-1.37$ & NS \\
\hline & HLC/outdoor & 340 & 230 & 110 & & & \\
\hline
\end{tabular}

$\mathrm{OR}$, odds ratio of indoor/outdoor human biting by Folonzo versus Kiribina; $\mathrm{Cl}$, confidence interval; $\mathrm{HLC}$, human landing catch.

coupled with comparably high rates of P. falciparum infection ( $>8 \%$ of indoor samples) emphasize the fact that Folonzo and Kiribina both are formidable malaria vectors in this part of Africa. The Kiribina form often outnumbered Folonzo. Yet, Folonzo was disproportionately represented in indoor versus outdoor resting samples and was more inclined to post-prandial endophily, while Kiribina was over-represented outdoors in pit shelters. This suggests that the overall An. funestus population is not uniformly exposed to indoor-based malaria interventions such as insecticide-treated nets and house spraying by residual insecticides, and that those indoor interventions are less effective against the Kiribina form.

There is precedence for chromosomal inversionassociated heterogeneity in mosquito resting behaviour in the West African savanna, uncovered by Coluzzi and colleagues through polytene chromosome analysis of $A n$. gambiae and An. arabiensis populations during the Garki Project in Nigeria [3,32]. Such behavioural heterogeneity was responsible for the failure to interrupt malaria transmission during the course of the Project, despite rigorous insecticide applications and simultaneous administration of anti-malarial drugs to the human population [33]. Indeed, there are hints that this same phenomenon has been witnessed previously with respect to An. funestus in the West African savanna, where Kiribina co-exists with Folonzo. In the absence of Kiribina in eastern and southern Africa, historical house spraying campaigns not only locally eliminated $A n$. funestus, but the effect was maintained for several years following the cessation of spraying, due to the apparent inability of $A n$. funestus to recolonize some areas [34]. Likewise, An. funestus was eliminated from humid forest and degraded forest areas in West Africa where malaria is meso- or hypo-endemic [34], an environment where Folonzo is predicted to dominate [16,35-37]. However, in the savannas of West Africa where malaria is holo- or hyperendemic, similar historical indoor spraying campaigns failed to eliminate the species [34]. Exophilic populations persisted which, despite marked anthropophily, continued to feed outdoors on cattle as well as humans, and also entered sprayed houses to bite humans, but escaped unharmed to rest outdoors. These exophilic populations likely represented what would now be recognized as the Kiribina form of An. funestus.

More recently, further epidemiologically significant behavioural heterogeneities in An. funestus from the same

Table 4 Host selection of Anopheles funestus chromosomal forms in Burkina Faso (2005-2007)

\begin{tabular}{|c|c|c|c|c|c|c|}
\hline Sample & Blood meal & Folonzo & Kiribina & OR & $0.95 \mathrm{Cl}$ & $P$ \\
\hline \multirow[t]{4}{*}{ ISC/indoors } & Human + Mixed & $191+21(212)$ & $232+11(243)$ & 2.81 & $1.30-6.07$ & 0.006 \\
\hline & Bovine & 9 & 29 & & & \\
\hline & Total & 221 & 272 & & & \\
\hline & $\mathrm{HBI}$ & $95.9 \%$ & $89.3 \%$ & & & \\
\hline \multirow[t]{4}{*}{ PIT/outdoors } & Human + Mixed & $2+1(3)$ & $13+25(38)$ & 1.44 & $0.42-4.95$ & $\mathrm{NS}^{*}$ \\
\hline & Bovine & 27 & 491 & & & \\
\hline & Total & 30 & 529 & & & \\
\hline & $\mathrm{HBI}$ & $10.0 \%$ & $7.2 \%$ & & & \\
\hline
\end{tabular}

*NS by Fisher Exact test. Chi-square not calculated due to an expected cell frequency below 5.

OR, odds ratio of human/bovine blood meal by Folonzo versus Kiribina; Cl, confidence interval; ISC, insecticide spray-sheet catch; PIT, Muirhead-Thomson pit-shelter [30]; Mixed, blood meal of human and bovine origin; HBI, human blood index. 
Table 5 Plasmodium falciparum sporozoite rate of Anopheles funestus chromosomal forms in Burkina Faso (2005-2007)

\begin{tabular}{lllllll}
\hline Sample & CSP & Folonzo & Kiribina & OR & $\mathbf{0 . 9 5} \mathbf{C l}$ & $\boldsymbol{P}$ \\
\hline ISC/indoor & + & 27 & 45 & 0.97 & $0.59-1.59$ & NS \\
& - & 291 & 469 & & & \\
& Total & 318 & 514 & & & \\
& $\% C S P+$ & $8.5 \%$ & $8.8 \%$ & & & \\
PIT/outdoor & + & 0 & 32 & 0.00 & $0.00-2.14$ & NS* $^{*}$ \\
& - & 61 & 1035 & & & \\
& Total & 61 & 1067 & & & \\
& $\% C S P+$ & $0.0 \%$ & $3.0 \%$ & & & \\
\hline
\end{tabular}

*NS by Fisher Exact test.

CSP, circumsporozoite protein; OR, odds ratio of sporozoite infection in Folonzo versus Kiribina; $\mathrm{Cl}$, confidence interval; ISC, insecticide spray-sheet catch; PIT, Muirhead-Thomson pit-shelter [30].

biogeographical area have been recognized following large-scale implementation of indoor-based vector control interventions. After mass deployment of insecticidetreated bed nets, the biting cycle of An. funestus shifted from its usual peak between 02:00 and 04:00 toward a later peak between dawn and early morning hours, when human hosts are less likely to be protected by nets [38]. Unfortunately, it is not known whether this behavioural shift was associated with a change in the chromosomal composition of the local An. funestus population.

The Folonzo and Kiribina chromosomal forms have been well characterized across several hundred kilometres and all ecozones of Burkina Faso [16,23]. However, their broader geographical distribution in Africa is poorly known. Certainly, they occur as far west as Senegal $[15,27,39]$. A recent study of sympatric populations of these forms, the first of its kind in Senegal, found stable co-existence of the forms across three successive breeding seasons and concluded, in accord with the present study, that Kiribina predominated, and rates of anthropophagy and sporozoite infection were comparable between forms, although both metrics were considerably lower in Senegal ( $\sim 30$ and $\sim 3 \%$, respectively) than they were in Burkina Faso [27]. Unfortunately, due to very low outdoor resting sample size (five total, of which only three could be identified chromosomally as Kiribina), indoor/outdoor resting behaviour was difficult to compare between forms, and thus, between studies. Cameroon is the most easterly country in which An. funestus chromosomal forms have been reported [36], but their vectorial heterogeneities (if any) are essentially uncharacterized. Available cytogenetic data suggest that these forms are largely allopatric in Cameroon, with Folonzo occurring in the mesic, forested south and Kiribina to the north in the dry savannas, except for a central contact zone at the forest-savanna transition, where stable sympatric co-existence of the two forms has not been clearly resolved [35-37]. In another parallel with the An. gambiae chromosomal forms, there is no evidence for the co-occurrence of An. funestus chromosomal forms in East Africa [40]; existing populations of An. funestus in eastern Africa are hypothesized to be allied with the Folonzo form [16], although that proposal has yet to be tested genetically.

Ample indication now exists of the practical importance of population structure and behavioural heterogeneities hidden within An. funestus, for malaria epidemiology and control in West Africa, if not beyond. In this light, the dearth of information about the wider geographic distribution and associated bionomics and vectorial parameters of the Folonzo and Kiribina forms is a problem that must be remedied as a matter of priority. The polytene chromosomes of An. funestus are considerably more difficult to spread and analyse than those of An. gambiae, a factor that has impeded past research on An. funestus. The demanding and specialized task of polytene chromosomebased identification, the restrictive sex and life stage from which favourable chromosomes are obtained, and the absence of any known DNA-based diagnostics to distinguish the chromosomal forms, all but prohibit deeper field investigation of Folonzo and Kiribina, particularly studies of their larval biology which is presumed to be a driver of their ecological and behavioural divergence. Genome sequencing of An. gambiae in 2002 [41], and the discovery of molecular forms of An. gambiae detectable by a simple PCR assay [26], greatly transformed understanding of the complexities of An. gambiae population structure and its impacts on malaria transmission. Recent whole genome sequencing and a newly available reference assembly for An. funestus [42] offer a platform that will support a more detailed understanding of An. funestus population structure across Africa, as well as an efficient means to discover genomic sequences potentially useful for molecular taxonomy of Folonzo and Kiribina.

For decades, patterns of chromosomal inversion polymorphism have guided discovery of population structure and even species boundaries hidden inside otherwise morphologically indistinguishable groups of anopheline mosquitoes i.e., [16,43-46]. Such an association of inversions with population substructure could be an incidental consequence of genetic drift owing to reduced gene flow, or the result of demographic history, but the observation that polymorphic inversions are often clinally distributed with respect to environmental gradients and subject to repeating seasonal fluctuations in frequency suggests that they are subject to strong selective forces [47]. In anopheline mosquitoes, as in many animal and plant species, chromosomal inversions are implicated in local adaptation to environmental heterogeneities [35,48-51]. To the extent that speciation may occur as a by-product of adaptive divergence, chromosomal inversions may also be instrumental in lineage splitting, as proposed by Coluzzi for 
anopheline mosquitoes [52]. That Kiribina and Folonzo forms are characterized by alternative arrangements of chromosomal inversions, and that these alternative arrangements shift in relative frequency according to geography, season, and larval habitat availability, suggests a direct role for chromosomal rearrangements in adaptation to heterogeneous and changing environments (see also $[35,50])$. Thus, beyond simply serving as markers for epidemiologically relevant population structure, alternative chromosomal arrangements some how condition different physiological and behavioural responses to the environment. A mechanistic understanding of what the adaptations are and how they evolved could prove instrumental in predicting how An. funestus may be capable of responding to future environmental challenges, including anthropogenic changes to climate and landscape, and exposure to new means of vector control.

\section{Competing interests}

The authors declare that they have no competing interests.

\section{Authors' contributions}

CC, NFS and NB conceived the study. WG performed the field collections. NFS provided logistical support throughout the study. WG performed the karyotyping. WG, CC and FL analysed results. WG, CC and NB wrote the manuscript. All authors read and approved the final manuscript.

\section{Acknowledgements}

We thank the inhabitants of Koubri and Kuiti for their collaboration, and the entomological team of CNRFP for their efforts during the course of this study. M A Yameogo, O Grushko and D Boccolini were instrumental in polytene chromosome analysis. This research was financially supported by grant R01 Al48842 from the US National Institutes of Health to NJB; re-entry grant A 41413 to WG from WHO/TDR; and WHO MIM/TDR grant 50090 to NFS.

\section{Author details}

${ }^{1}$ Centre National de Recherche et de Formation sur le Paludisme, Ouagadougou 01 BP 2208, Burkina Faso. ²Department of Applied and Computational Mathematics and Statistics, University of Notre Dame, Notre Dame, IN 46556, USA. ${ }^{3}$ Eck Institute for Global Health, Department of Biological Sciences, University of Notre Dame, Notre Dame, IN 46556, USA ${ }^{4}$ Institut de Recherche pour le Développement (IRD), Unité Mixte de Recherche MIVEGEC (UM1, UM2, CNRS 5290, IRD 224), Centre IRD de Montpellier 956, Avenue Agropolis BP 64501, Montpellier Cedex 5 34394, France.

Received: 21 January 2014 Accepted: 20 February 2014

Published: 24 February 2014

\section{References}

1. Sinka ME, Bangs MJ, Manguin S, Rubio-Palis Y, Chareonviriyaphap T, Coetzee M, Mbogo CM, Hemingway J, Patil AP, Temperley WH, Gething PW, Kabaria CW, Burkot TR, Harbach RE, Hay SI: A global map of dominant malaria vectors. Parasit Vectors 2012, 5:69.

2. Gillies MT, De Meillon B: The Anophelinae of Africa South of the Sahara. 2nd edition. Johannesburg: South African Institute for Medical Research; 1968.

3. Coluzzi M, Sabatini A, Petrarca V, Di Deco MA: Chromosomal differentiation and adaptation to human environments in the Anopheles gambiae complex. Trans R Soc Trop Med Hyg 1979, 73:483-497.

4. White BJ, Collins FH, Besansky NJ: Evolution of Anopheles gambiae in relation to humans and malaria. Annu Rev Ecol Evol Syst 2011, 42:111-132.

5. Garros C, Harbach RE, Manguin S: Systematics and biogeographical implications of the phylogenetic relationships between members of the Funestus and Minimus groups of Anopheles (Diptera: Culicidae). J Med Entomol 2005, 42:7-18.
6. Harbach RE: The classification of genus Anopheles (Diptera: Culicidae): a working hypothesis of phylogenetic relationships. Bull Entomol Res 2004 94:537-553

7. Coetzee M, Koekemoer LL: Molecular systematics and insecticide resistance in the major African malaria vector Anopheles funestus. Annu Rev Entomol 2013, 58:393-412.

8. Spillings BL, Brooke BD, Koekemoer LL, Chiphwanya J, Coetzee M, Hunt RH: A new species concealed by Anopheles funestus Giles, a major malaria vector in Africa. Am J Trop Med Hyg 2009, 81:510-515.

9. Hackett BJ, Gimnig J, Guelbeogo W, Costantini C, Koekemoer LL, Coetzee M, Collins FH, Besansky NJ: Ribosomal DNA internal transcribed spacer (ITS2) sequences differentiate Anopheles funestus and An. rivulorum, and uncover a cryptic taxon. Insect Mol Biol 2000, 9:369-374.

10. Cohuet A, Simard F, Toto J-C, Kengne P, Coetzee M, Fontenille D: Species identification within the Anopheles funestus group of malaria vectors in Cameroon and evidence for a new species. Am J Trop Med Hyg 2003 69:200-205.

11. Koekemoer LL, Misiani EA, Hunt RH, Kent RJ, Norris DE, Coetzee M: Cryptic species within Anopheles longipalpis from southern Africa and phylogenetic comparison with members of the An. funestus group. Bull Entomol Res 2009, 99:41-49.

12. Sinka ME, Bangs MJ, Manguin S, Coetzee M, Mbogo CM, Hemingway J, Patil AP, Temperley WH, Gething PW, Kabaria CW, Okara RM, Van Boeckel T, Godfray HC, Harbach RE, Hay SI: The dominant Anopheles vectors of human malaria in Africa, Europe and the Middle East: occurrence data, distribution maps and bionomic precis. Parasit Vectors 2010, 3:117.

13. Sharakhov I, Braginets O, Grushko O, Cohuet A, Guelbeogo WM, Boccolini D, Weill M, Costantini C, Sagnon N, Fontenille D, Yan G, Besansky NJ: A microsatellite map of the African human malaria vector Anopheles funestus. J Hered 2004, 95:29-34.

14. Green CA, Hunt RH: Interpretation of variation in ovarian polytene chromosomes of Anopheles funestus Giles, A. parensis Gillies, and A. aruni? Genetica 1980, 51:187-195.

15. Lochouarn L, Dia I, Boccolini D, Coluzzi M, Fontenille D: Bionomical and cytogenetic heterogeneities of Anopheles funestus in Senegal. Trans $R$ Soc Trop Med Hyg 1998, 92:607-612.

16. Costantini C, Sagnon NF, Ilboudo-Sanogo E, Coluzzi M, Boccolini D: Chromosomal and bionomic heterogeneities suggest incipient speciation in Anopheles funestus from Burkina Faso. Parassitologia 1999, 41:595-611.

17. Guelbeogo WM, Grushko O, Boccolini D, Ouedraogo PA, Besansky NJ, Sagnon NF, Costantini C: Chromosomal evidence of incipient speciation in the Afrotropical malaria mosquito Anopheles funestus. Med Vet Entomol 2005, 19:458-469.

18. Michel AP, Ingrasci MJ, Schemerhorn BJ, Kern M, Le Goff G, Coetzee M, Elissa N, Fontenille D, Vulule J, Lehmann T, Sagnon N, Costantini C, Besansky NJ: Rangewide population genetic structure of the African malaria vector Anopheles funestus. Mol Ecol 2005, 14:4235-4248.

19. Koekemoer LL, Kamau L, Garros C, Manguin S, Hunt RH, Coetzee M: Impact of the Rift Valley on restriction fragment length polymorphism typing of the major African malaria vector Anopheles funestus (Diptera: Culicidae). J Med Entomol 2006, 43:1178-1184.

20. Choi KS, Koekemoer LL, Coetzee M: Population genetic structure of the major malaria vector Anopheles funestus s.s. and allied species in southern Africa. Parasit Vectors 2012, 5:283.

21. Coluzzi M, Petrarca V, DiDeco MA: Chromosomal inversion intergradation and incipient speciation in Anopheles gambiae. Boll Zool 1985, 52:45-63.

22. Guelbeogo WM, Sagnon N, Grushko O, Yameogo MA, Boccolini D, Besansky NJ, Costantini C: Seasonal distribution of Anopheles funestus chromosomal forms from Burkina Faso. Malar J 2009, 8:239.

23. Michel AP, Grushko O, Guelbeogo WM, Lobo NF, Sagnon N, Costantini C, Besansky NJ: Divergence with gene flow in Anopheles funestus from the Sudan Savanna of Burkina Faso, West Africa. Genetics 2006, 173:1389-1395.

24. Michel AP, Guelbeogo WM, Grushko O, Schemerhorn BJ, Kern M, Willard MB, Sagnon N, Costantini C, Besansky NJ: Molecular differentiation between chromosomally defined incipient species of Anopheles funestus. Insect Mol Biol 2005, 14:375-387.

25. Coetzee M, Hunt RH, Wilkerson R, Della Torre A, Coulibaly MB, Besansky NJ: Anopheles coluzzii and Anopheles amharicus, new members of the Anopheles gambiae complex. Zootaxa 2013, 3619:246-274. 
26. della Torre A, Fanello C, Akogbeto M, Dossou-yovo J, Favia G, Petrarca V, Coluzzi M: Molecular evidence of incipient speciation within Anopheles gambiae s.s. in West Africa. Insect Mol Biol 2001, 10:9-18.

27. Dia I, Sagnon N, Guelbeogo MW, Diallo M: Bionomics of sympatric chromosomal forms of Anopheles funestus (Diptera: Culicidae). J Vector Ecol 2011, 36:343-347.

28. Gillies MT, Coetzee M: A Supplement to the Anophelinae of Africa South of the Sahara. Johannesburg: The South African Institute for Medical Research; 1987.

29. Della Torre A: Polytene chromosome preparation from anopheline mosquitoes. In Molecular Biology of Disease Vectors: A Methods Manual. Edited by Crampton JM, Beard CB, Louis C. London: Chapman \& Hall; 1997:329-336.

30. Service MW: Mosquito Ecology. Field Sampling Methods. 2nd edition. London: Elsevier Applied Science Publishers, Ltd; 1993.

31. Bland JM, Altman DG: Statistics notes. The odds ratio. Br Med J 2000 320:1468.

32. Powell JR, Petrarca V, della Torre A, Caccone A, Coluzzi M: Population structure, speciation, and introgression in the Anopheles gambiae complex. Parassitologia 1999, 41:101-113.

33. Molineaux L, Grammicia G: The Garki Project. Research on the Epidemiology and Control of Malaria in the Sudan Savanna of West Africa. Geneva, Switzerland: World Health Organization; 1980.

34. Zahar AR: Vector Bionomics in the Epidemiology and Control of Malaria. Geneva, Switzerland: World Health Organization; 1985.

35. Ayala D, Fontaine MC, Cohuet A, Fontenille D, Vitalis R, Simard F: Chromosomal inversions, natural selection and adaptation in the malaria vector Anopheles funestus. Mol Biol Evol 2011, 28:745-758.

36. Cohuet A, Dia I, Simard F, Raymond M, Rousset F, Antonio-Nkondjio C, Awono-Ambene PH, Wondji CS, Fontenille D: Gene flow between chromosomal forms of the malaria vector Anopheles funestus in Cameroon, Central Africa, and its relevance in malaria fighting. Genetics 2005, 169:301-311.

37. Dia I, Boccolini D, Antonio-Nkondjio C, Costantini C, Fontenille D: Chromosomal inversion polymorphism of Anopheles funestus from forest villages of South Cameroon. Parassitologia 2000, 42:227-229.

38. Moiroux N, Gomez MB, Pennetier C, Elanga E, Djenontin A, Chandre F, Djegbe I, Guis H, Corbel V: Changes in Anopheles funestus biting behavior following universal coverage of long-lasting insecticidal nets in Benin. J Inf Dis 2012, 206:1622-1629.

39. Dia I, Lochouarn L, Boccolini D, Costantini C, Fontenille D: Spatial and temporal variations of the chromosomal inversion polymorphism of Anopheles funestus in Senegal. Parasite 2000, 7:179-184.

40. Kamau L, Hunt R, Coetzee M: Analysis of the population structure of Anopheles funestus (Diptera: Culicidae) from western and coastal Kenya using paracentric chromosomal inversion frequencies. J Med Entomol 2002, 39:78-83.

41. Holt RA, Subramanian GM, Halpern A, Sutton GG, Charlab R, Nusskern DR, Wincker P, Clark AG, Ribeiro JM, Wides R, Salzberg SL, Loftus B, Yandell M, Majoros WH, Rusch DB, Lai Z, Kraft CL, Abril JF, Anthouard V, Arensburger P, Atkinson PW, Baden $\mathrm{H}$, de Berardinis V, Baldwin D, Benes V, Biedler J, Blass C, Bolanos R, Boscus D, Barnstead M, et al: The genome sequence of the malaria mosquito Anopheles gambiae. Science 2002, 298:129-149.

42. Neafsey DE, Christophides GK, Collins FH, Emrich SJ, Fontaine MC, Gelbart W, Hahn MW, Howell PI, Kafatos FC, Lawson D, Muskavitch MA, Waterhouse RM, Williams $\sqcup$, Besansky NJ: The evolution of the Anopheles 16 genomes project. G3 2013, 3:1191-1194.

43. Subbarao SK: Anopheles Species Complexes in South and South-East Asia. SEARO Technical Publication No. 57. New Delhi: World Health Organization, Regional Office for South-East Asia; 2007.

44. Toure YT, Petrarca V, Traore SF, Coulibaly A, Maiga HM, Sankare O, Sow M, DiDeco MA, Coluzzi M: The distribution and inversion polymorphism of chromosomally recognized taxa of the Anopheles gambiae complex in Mali, West Africa. Parassitologia 1998, 40:477-511.

45. Coluzzi M, Sabatini A, Della Torre A, Di Deco MA, Petrarca V: A polytene chromosome analysis of the Anopheles gambiae species complex. Science 2002, 298:1415-1418.

46. Kitzmiller JB, Frizzi G, Baker RH: Evolution and speciation within the Maculipennis Complex of the genus Anopheles. In Genetics of Insect Vectors of Disease. Edited by Wright JW, Pal R. Amsterdam: Elsevier; 1967.
47. Hoffmann AA, Rieseberg LH: Revisiting the impact of inversions in evolution: from population genetic markers to drivers of adaptive shifts and speciation? Ann Rev Ecol Evol Syst 2008, 39:21-42.

48. Kirkpatrick M, Barton N: Chromosome inversions, local adaptation and speciation. Genetics 2006, 173:419-434.

49. Kirkpatrick M: How and why chromosome inversions evolve. PLoS Biol 2010, 8(9):e1000501. doi:10.1371/journal.pbio.1000501.

50. Ayala D, Guerrero RF, Kirkpatrick M: Reproductive isolation and local adaptation quantified for a chromosome inversion in a malaria mosquito. Evolution 2013, 67:946-958.

51. Cheng C, White BJ, Kamdem C, Mockaitis K, Costantini C, Hahn MW, Besansky NJ: Ecological genomics of Anopheles gambiae along a latitudinal cline: a population-resequencing approach. Genetics 2012 190:1417-1432.

52. Coluzzi M: Spatial distribution of chromosomal inversions and speciation in anopheline mosquitoes. In Mechanisms of Speciation. Edited by Barigozzi C. New York: Alan R. Liss, Inc; 1982:143-153.

doi:10.1186/1475-2875-13-65

Cite this article as: Guelbeogo et al: Behavioural divergence of sympatric Anopheles funestus populations in Burkina Faso. Malaria Journal 2014 13:65.

\section{Submit your next manuscript to BioMed Central and take full advantage of:}

- Convenient online submission

- Thorough peer review

- No space constraints or color figure charges

- Immediate publication on acceptance

- Inclusion in PubMed, CAS, Scopus and Google Scholar

- Research which is freely available for redistribution 